\title{
Paired related homeobox protein 1 regulates PDGF-induced chemotaxis of hepatic stellate cells in liver fibrosis
}

\author{
Jin Gong ${ }^{1}$, Jian Han ${ }^{1}$, Jiayi He², Jingmei Liu ${ }^{1}$, Ping Han ${ }^{1}$, Yunwu Wang ${ }^{1}$, Mengke Li ${ }^{1}$, Dongxiao Li ${ }^{1}$, Xiangming Ding ${ }^{1}$, \\ Zhipeng Du', Jiazhi Liao ${ }^{1,3}$ and Dean Tian ${ }^{1,3}$
}

Activation of the platelet-derived growth factor (PDGF)/PDGF beta receptor (PDGF $\beta$ R) axis has a critical role in liver fibrosis. However, the mechanisms that regulate the PDGF signaling are yet to be elucidated. The present study demonstrates that paired related homeobox protein 1 (Prrx1) is involved in PDGF-dependent hepatic stellate cell (HSCs) migration via modulation of the expression of metalloproteinases MMP2 and MMP9. PDGF elevated the level of Prrx1 through the activation of ERK/Sp1 and PI3K/Akt/Ets1 pathways. In vivo, an adenoviral-mediated Prrx1 shRNA administration attenuated liver fibrosis in thioacetamide-induced fibrotic models. These studies reveal a role of Prrx 1 as a modulator of PDGF-dependent signaling in HSCs, and inhibiting its expression may offer a therapeutic approach for hepatic fibrosis. Laboratory Investigation (2017) 97, 1020-1032; doi:10.1038/labinvest.2017.65; published online 24 July 2017

Progressive liver fibrosis is a common consequence of chronic hepatic injury and is characterized by excessive accumulation of extracellular matrix (ECM). ${ }^{1}$ The activation of hepatic stellate cells (HSCs) is the key event during hepatic fibrogenesis. As a response to continued liver damage, HSCs transform from a quiescent state into fibrogenic, proliferative, contractile, and $\alpha$-smooth muscle actin ( $\alpha$-SMA)-positive myofibroblast-like phenotype. This transformation is also accompanied by an abundant production of extracellular matrix (ECM) and secretion of high levels of cytokines, such as transforming growth factor- $\beta 1$ (TGF- $\beta 1$ ) and plateletderived growth factor (PDGF)., ${ }^{2,3}$

PDGF is a potent mitogen and chemotactic cytokine that is frequently elevated in a fibrotic liver. ${ }^{4-6}$ Previous studies have shown that the overexpression of PDGFs could drive the proliferation, motility, and chemotaxis of HSCs, ${ }^{5,6}$ whereas the loss of PDGFR or PDGF $\beta$ leads to embryonic lethality in mice. ${ }^{7,8}$ In addition, the specific depletion of PDGF- $\beta$ R in HSCs reduces the liver injury and fibrosis in mice. ${ }^{9}$

The pair-related homeobox transcription factor, Prrxl, has two alternative splicing isoforms, Prrxla and Prrxlb. These isoforms are primarily different at the C-terminal end and interact with other factors to enhance the specific DNA binding activity in a similar manner. ${ }^{10,11}$ Under physiological conditions, Prrxl has crucial roles in embryonic development. Prrxl ${ }^{-/-}$mice suffer perinatal death along with craniofacial defects and limb shortening. ${ }^{12-14}$ Prrxl is also involved in the self-renewal in neural stem cells and pancreatic regeneration, ${ }^{15,16}$ and its expression promotes tenascin C-dependent fibroblast migration. ${ }^{17}$ Recent evidence show that Prrxl can induce the epithelial-mesenchymal transition (EMT) in pancreatic and breast cancer cells. Furthermore, the knockdown of Prrxl manifests stem celllike features in cancer cells. ${ }^{16,18}$ Prrxl is also implicated in the regulation of mesenchymal cell fate. Previous studies demonstrated that Prrxl was highly upregulated in the fibrotic liver and was responsible for the synthesis of procollagen and transforming growth factor beta 3 (TGFB3) in the activated HSCs. ${ }^{19,20}$

In this study, we demonstrated for the first time that Prrx1 acts as a critical downstream molecule in PDGF signaling pathway and has a major role in the recruitment of PDGFdependent HSCs. These discoveries provide new insights into the mechanisms of PDGF-regulated processes; also it might serve as a novel target for the therapeutic intervention in fibrotic liver.

\footnotetext{
'Department of Gastroenterology, Tongji Hospital of Tongji Medical College, Huazhong University of Science and Technology, Wuhan, China and ${ }^{2}$ Department of Pediatrics, Tongji Hospital of Tongji Medical College, Huazhong University of Science and Technology, Wuhan, China Correspondence: Professor D Tian, MD, PhD or Professor J Liao, MD, PhD, Department of Gastroenterology, Tongji Hospital of Tongji Medical College, Huazhong University of Science and Technology, Wuhan 430030, China

${ }^{3}$ These authors contributed equally to this work.

Received 16 January 2017; revised 16 April 2017; accepted 3 May 2017
} 


\section{MATERIALS AND METHODS \\ Reagents}

Human recombinant PDGF-BB was purchased from Peprotech (Rocky Hill, CT, USA). Compounds such as U0126 and LY294002 were obtained from Sigma (St Louis, MO, USA). Fetal bovine serum (FBS) was purchased from Gibco (Carlsbad, CA, USA) and fibronectin from Corning (New York, NY, USA).

\section{Isolation and Culture of HSCs}

Primary HSCs were isolated from male Sprague Dawley rats (SD; 200-250 g) by the pronase-collagenase perfusion in situ before density gradient centrifugation on an OptiPrep gradient, as described previously. ${ }^{21}$ The primary cells were cultured in Dulbecco's modified Eagle's medium (DMEM) supplemented with $20 \%$ FBS. The passaged cells were grown in DMEM with $10 \%$ of FBS; the rat primary HSCs (R-HSC) were used for up to four passages. The human hepatic stellate cell line, LX-2, obtained from the Institute of Liver Diseases, Tongji Hospital of Tongji Medical College, Huazhong University of Science and Technology, (Wuhan, China) was cultured in DMEM with 10\% FBS.

\section{siRNA, Plasmids, Virus, and Cell Transfection}

Small interfering RNA (siRNA) duplexes targeting Prrx1, Sp1, and Ets1, as well as scrambled siRNA (NC; Supplementary Table 1), were synthesized by RiboBio (Guangzhou, China). Human Prrxla (CMV-MCS-3FLAG-SV40-Neomycin) and Prrxlb plasmids, the control vector, an adenoviral vector containing shRNA targeting mouse Prrxl (AdshPrrxl, targeted sites: $5^{\prime}$-CCAACAGCATTGCCAACCT- ${ }^{\prime}$ ), and the control adenovirus (AdshNC) were purchased from Genechem (Shanghai, China). The HSCs were transfected with indicated siRNA or plasmids using Lipofectamine 2000 reagent (Carlsbad, CA, USA) according to the standard protocols, and the knockdown efficiency was assessed by realtime PCR and western blot. All the assays were performed at 72-96 h after transfection.

\section{Western Blot and Co-Immunoprecipitations}

Following standard electrophoresis, the membranes were probed overnight at $4{ }^{\circ} \mathrm{C}$ with primary antibodies listed in Supplementary Table 2. Subsequently, the membranes were incubated with anti-mouse or -rabbit IgG (1:3000; Abbkine, CA, USA), and the signals were detected using an ECL assay kit (Amersham, Buckinghamshire, UK).

For co-immunoprecipitations, the cells were lysed with $1 \%$ NP40 NET buffer (Promoter Company, China), the supernatants were incubated overnight at $4{ }^{\circ} \mathrm{C}$ with protein G-Sepharose beads (Sigma) conjugated with rabbit antiFlagor anti-IgG antibody, and then the samples were prepared for western blot assay. For western blot, the membrane was incubated overnight at $4{ }^{\circ} \mathrm{C}$ with primary antibodies Sp1, Ets1, Flag, and Prrx1, followed by anti-mouse or -rabbit IgG
(1:3000; Sigma, CA, USA), and the signals were detected with an ECL assay kit (Amersham).

\section{Patients Liver Samples}

Informed consent was obtained from the tissue donors before collecting a total of 20 fibrotic liver samples from surgical resections in patients with liver hemangioma at the Tongji Hospital in 2015. The procedure of collection of the human samples was approved by the Ethics Committee of the Tongji Hospital, Huazhong University of Science and Technology, and the study was conducted according to the Declaration of Helsinki principles. The liver tissues were fixed in $4 \%$ buffered paraformaldehyde and embedded in paraffin for immunostaining.

Animal Fibrosis Models and Adenoviral Delivery In Vivo $\mathrm{CCl}_{4}$-induced-fibrosis models: male C57 mice, 7-10-weekold, were intraperitoneally $(0.5 \mu \mathrm{l} / \mathrm{g}$ of body weight, two times/week) injected with $25 \% \mathrm{CCl}_{4}$ (carbon tetrachloride) to induce chronic liver injury, whereas the control mice were administered only olive oil. The injections were continued for 0,4 , and 8 weeks, respectively $(n=5)$ to establish the model for fibrosis with different degrees of severity.

TAA-induced-fibrosis models: male Sprague Dawley (SD) rats, weighing about $300 \mathrm{~g}$, were purchased from the Department of Experimental Animals of Tongji Medical College. The animals $(n=8)$ were treated with thioacetamide (TAA solubilized in saline, $200 \mathrm{mg} / \mathrm{kg}$ body weight; Ding Guo, China) by the intraperitoneal injection two times/week for 4 , 8 , or 12 weeks, respectively, to induce chronic liver injury; the rats were killed 2 days after the final dose. The control group received an equivalent amount of saline alone $(n=8)$.

Adenoviral delivery in vivo: hepatic fibrosis was induced in rats by intraperitoneal injection of TAA $(200 \mathrm{mg} / \mathrm{kg}$ body weight) two times/week for 6 weeks. Subsequently, each animal received $5 \times 10^{9} \mathrm{PFU}$ of Prrxl shRNA (AdshPrrx1) or control adenovirus vector (AdshNC) via the tail vein, one time/week and was repetitively administered TAA for 2 weeks.

The liver tissues and serum were either snap-frozen at $-80^{\circ} \mathrm{C}$ or fixed in $4 \%$ buffered paraformaldehyde for immunostaining. All animal studies were performed in accordance with the national and international guidelines. The protocol was approved by the Ethics Committee of Animal Experiments of Tongji Medical College and monitored by the Department of Experimental Animals of the Tongji Medical College.

\section{Migration Assay}

HSCs transfected with Prrxl siRNA in the presence or absence of PDGF-BB $(20 \mathrm{ng} / \mathrm{ml})$ for $24 \mathrm{~h}$ were resuspended in low-serum medium and seeded in the upper chamber $(20000$ cells/well) of the polycarbonate membrane transwell inserts ( $8 \mu \mathrm{m}$ pore size; Corning, China). The bottom chambers of the system were filled with serum-free medium containing PDGF $(10 \mathrm{ng} / \mathrm{ml})$. After incubation for $48 \mathrm{~h}$, the 
a
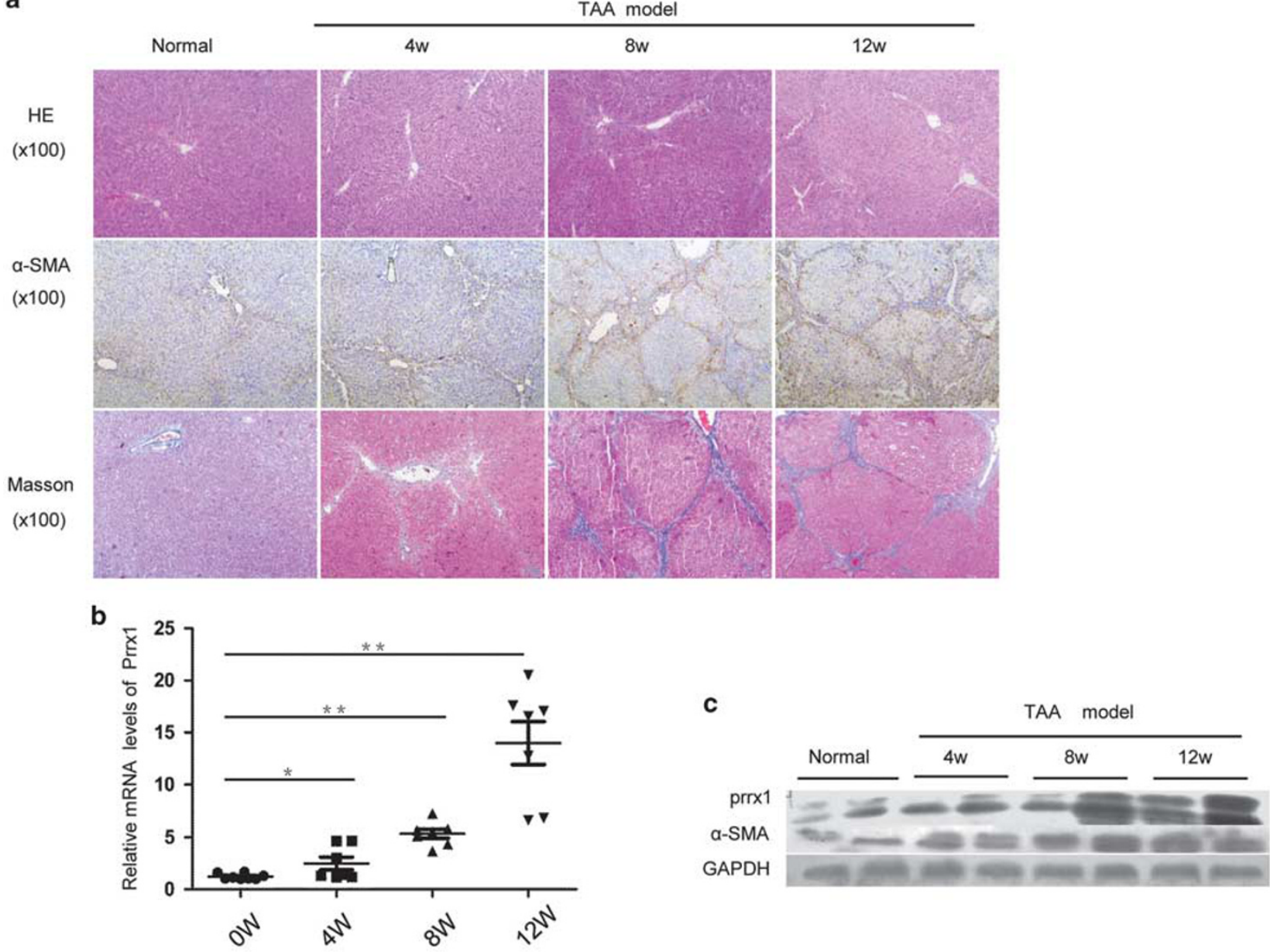

d

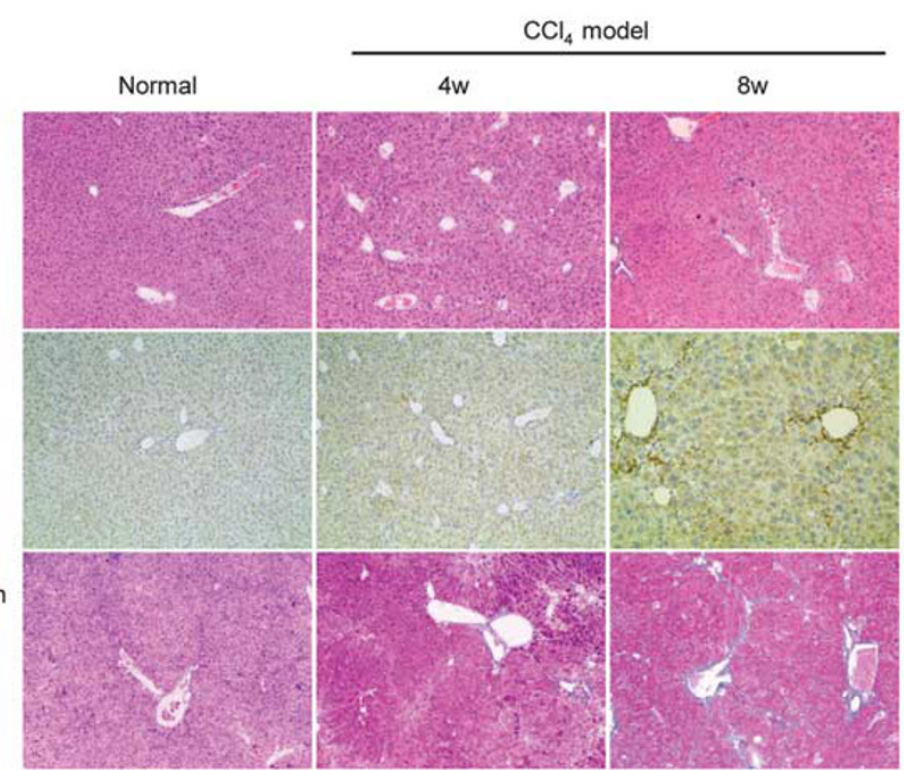

e

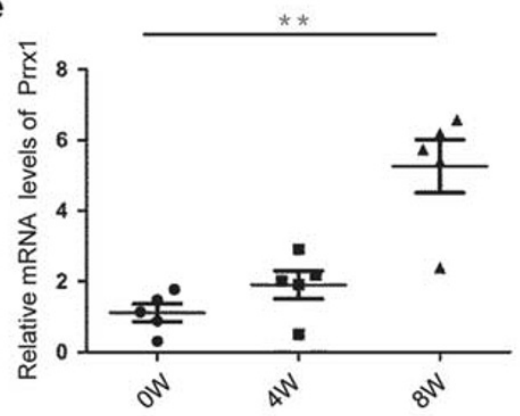

$\mathbf{f}$

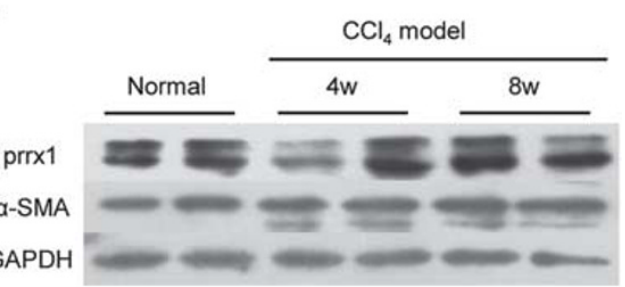

Figure 1 Increased expression of Prrx1 in fibrotic models. (a) The expression of $a$-SMA was assessed by immunohistochemistry. HE and Masson's trichrome staining were used to examine the pathological alterations and collagen deposition in TAA-treated rats. (b and $\mathbf{c})$ The mRNA and protein levels of Prrx 1 and $a$-SMA in the fibrotic livers from TAA-treated rats were detected by real-time PCR $(n=7)$ and western blot $(n=2)$ in each group, respectively. (d) $\mathrm{HE}$ and Masson's trichrome staining were used to examine the pathological alterations and collagen deposition in $\mathrm{CCl}_{4}$-treated mice. (e and $\mathbf{f}$ ) The mRNA and protein levels of Prrx 1 and $a$-SMA in the fibrotic livers from $\mathrm{CCl}_{4}$-treated rats were detected by real-time PCR $(n=5)$ and western blot $(n=2)$ in each group, respectively. GAPDH was used as a loading control. ${ }^{*} P<0.05,{ }^{* *} P<0.01$ vs normal control group by Student's $t$-test. 
polycarbonate filter was removed and the cells that had migrated to the lower chamber were stained with crystal violet and counted using a microscope; four fields/well were recorded, and the mean number of cells/field was calculated.

\section{In Vitro Wound-Repair Assay}

The LX-2 cells were seeded in six-well plates overnight to allow $50 \%$ confluency. Subsequently, the cells were transfected with Prrxl siRNA and control siRNA using Lipofectamine 2000 according to the manufacturer's instructions. After $24 \mathrm{~h}$, the growth medium was replaced with serum-free medium supplemented with human recombinant PDGF-BB $(20 \mathrm{ng} / \mathrm{ml})$. For wound assays, the cell monolayer was scratched using a sterile $200 \mu \mathrm{l}$ tip after another $24 \mathrm{~h}$. The cell growth in the denuded area was assessed after $24 \mathrm{~h}$, and six images from each sample were captured $(\times 10$ magnification). Cell migration was quantified as the percentage of the wound-healed area.

\section{Adhesion Experiments}

The 96-well plates were coated with fibronectin $(10 \mu \mathrm{g} / \mathrm{ml})$ at $37^{\circ} \mathrm{C}$ overnight. The plates were washed with PBS, blocked with $5 \%$ BSA for $1 \mathrm{~h}$ at room temperature, and washed again with PBS. NC- or Prrx1 siRNA-transfected LX-2 cells or primary rat HSCs (4000 cells/well) were incubated in serumfree medium for $2 \mathrm{~h}$ at $37^{\circ} \mathrm{C}$. The non-adherent cells were removed, and the others were fixed with $4 \%$ paraformaldehyde for $15 \mathrm{~min}$, stained with DAPI, and images captured by a fluorescent microscope for analysis. In addition, we also assessed the adherent cells by incubating them with CCK- 8 reagent (Promoter Biotechnology, Wuhan, China) for $3 \mathrm{~h}$, following which, the OD was measured on a multimode reader.

\section{Statistical Analysis}

All the experiments were performed in triplicate unless otherwise specified. The data were presented as mean \pm s.d. Statistical analyses were performed by Student's $t$-test using Prism 5.0 (GraphPad Software, La Jolla, CA, USA). A value of $P<0.05$ was considered statistically significant.

\section{RESULTS}

\section{Prrx1 Expression in HSC Increases During Liver Injury} and Correlates with the Level of Fibrosis

To confirm the role of Prrxl in liver fibrosis, we assessed the expression of Prrx1 in two animal models of induced fibrosis: the rats treated with TAA and the mice were treated with $\mathrm{CCl}_{4}$. The mRNA and protein levels of Prrxl were upregulated in TAA-induced fibrotic livers as compared with the normal livers (Figure $1 \mathrm{a}-\mathrm{c}$ ). A similar pattern was also observed in chronic $\mathrm{CCl}_{4}$-induced liver fibrosis in mouse liver where the Prrxl expression appeared higher than in the uninjured mouse liver (Figure 1d-f).

To further investigate the localization of Prrx1 in injured livers, we performed immunofluorescence double staining.
The results revealed that activated HSCs $\left(\alpha-\mathrm{SMA}^{+}\right.$cells $)$ strongly expressed Prrx1 in surgically resected fibrotic liver samples and that of TAA-treated rats (Figure 2a and Supplementary Figure 1). In order to confirm the identity of the cells, we isolated quiescent rat HSCs and stimulated them in culture. These activated HSCs exhibited a bright staining of Prrxl in the nucleus (Figure 2b), suggesting that Prrxl could have a role in HSC activation.

\section{PDGF-BB Induces Prrx1 Expression in HSCs}

PDGF-BB is primarily a mitogen. ${ }^{2}$ The increased expression of $\mathrm{PDGF} \beta$ receptor (PDGF- $\beta \mathrm{R}$ ) is a hallmark of HSC activation. ${ }^{22}$ Thus, we explored whether PDGF-BB could regulate Prrxl expression in HSCs. A dose-dependent regulation revealed that mRNA and protein levels of Prrxl increased noticeably after treatment with PDGF-BB at $20 \mathrm{ng} / \mathrm{ml}$ for $48 \mathrm{~h}$ (Figure $3 \mathrm{a}$ and b). Furthermore, PDGF-BB promoted the expression of Prrxl in a time-dependent manner, and this increase was sustained for $72 \mathrm{~h}$ (Figure $3 \mathrm{c}$ and $\mathrm{d}$ ). In addition, the expressions of Sp1 and p-Ets1 increased in response to PDGF-BB treatment and were significantly correlated with the expression of Prrxl (Figure $3 \mathrm{c}$ and $\mathrm{d}$ ). These data indicated that PDGF-BB directly activates the Prrxl expression at the transcriptional level.

\section{PDGF-BB Upregulates Prrx1 Expression Through PI3K and ERK Pathways}

Next, we sought to investigate the molecular mechanisms underlying the PDGF-BB-induced Prrxl expression. The canonical downstream effectors for PDGF $\beta$ R-dependent trophic actions are Akt and ERK. ${ }^{23}$ The pretreatment with ERK1/2 inhibitor (U0126) or PI3K inhibitor (LY294002) significantly blocked the PDGF-induced upregulation of Prrx $1 \mathrm{mRNA}$ and protein level (Figure $4 \mathrm{a}$ and b). Notably, the levels of Sp1 and phosphorylated Ets1 were altered, when ERK and Akt pathways were inactivated, respectively (Figure $4 \mathrm{~b}$ ). Hence, we revealed that PDGF-BB induces Prrx1 expression in HSCs via PI3K/Akt and ERK-dependent mechanisms.

\section{Transcription Factor Sp1 and Ets1 have Crucial Roles in Prrx1 Expression Mediated by PDGF-BB}

A previous study has demonstrated that PDGF-BB could promote Sp1 expression, nuclear localization, and phosphorylation to increase its transcriptional activity. ${ }^{24}$ Reportedly, Ets1, as the downstream effector of PI3K signaling pathway, is upregulated by PDGF-BB. ${ }^{25}$ Our results confirmed that PDGF-BB upregulated the expressions of Sp1 and p-Ets1 similar to that of the Prrxl level. Therefore, we examined the effect of silencing Sp1 and Ets1 on PDGF-BB-induced Prrx1 expression. The expressions of Sp1 and Ets1 were silenced in LX-2 cells, respectively, before treating with vehicle or PDGFBB. The results showed that the knockdown of Sp1 or Ets1 dramatically ablated the PDGF-BB-induced Prrx1 mRNA and 
a Human fibrotic tissue $400 x$

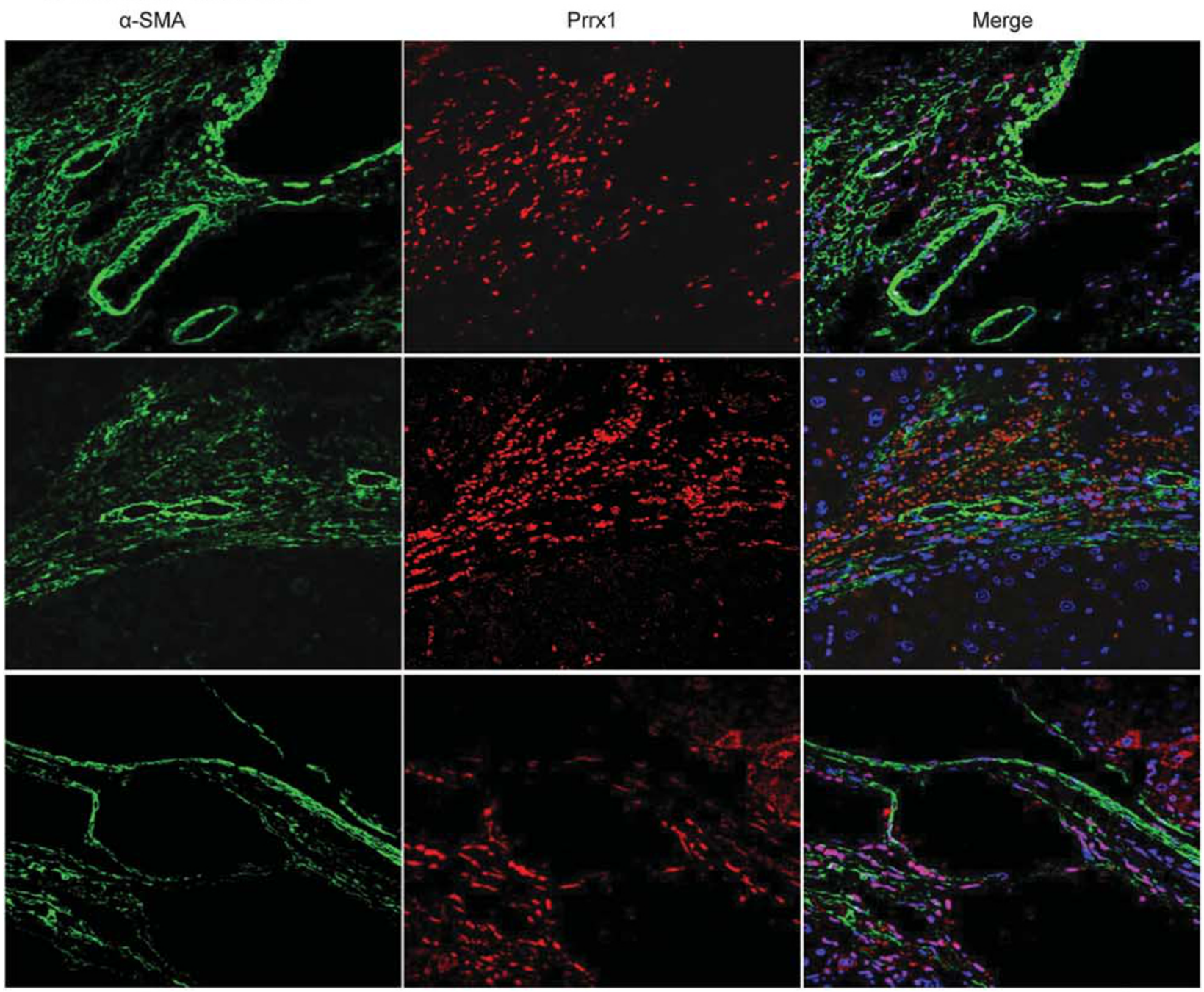

b R-HSC $200 x$

$1 d$

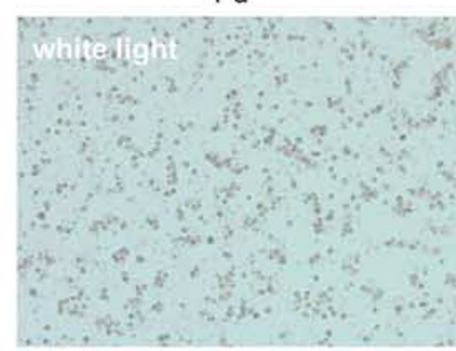

Autofluroscence
Prrx1
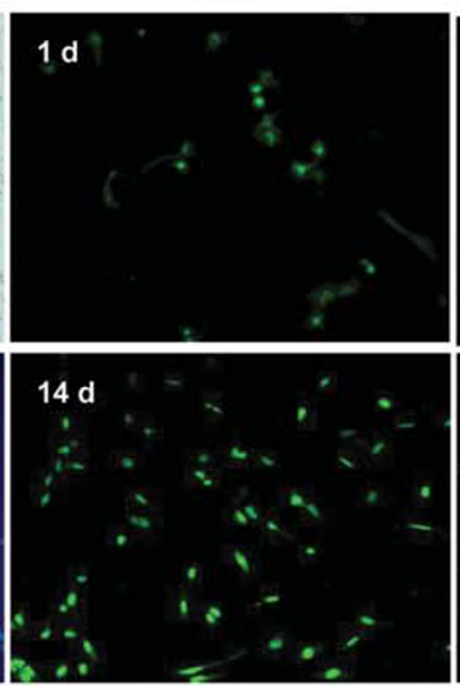

a-SMA

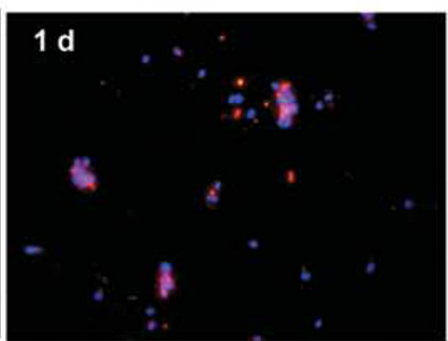

$14 \mathrm{~d}$

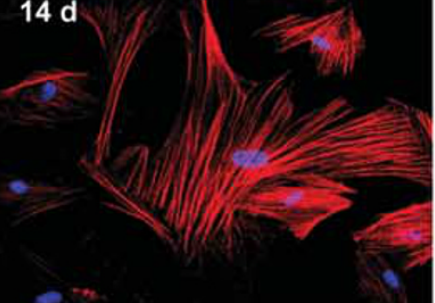


protein expression. Also, MMP2 and cyclinD1 were downregulated (Figure $4 \mathrm{c}-\mathrm{f}$ ). Compared with targeting Sp1 or Ets1 separately, combined siRNA targeting Sp1 and Ets1 did not further decrease the expression Prrx1 significantly, so it could be possible that the two pathway have a synergistic effect on Prrx1 expression Prrx1 mRNA level (Figure 4g). In addition, restoring the expression of Prrxl partially recovered the expression of MMP2 and cyclinD1 in Sp1 or Ets1 siRNAtransfected LX-2 cells (Figure $4 \mathrm{~h}$ and i). These data demonstrated that Sp1 and Ets1 are required for the PDGFBB-mediated Prrxl transcription activation.

\section{Role of Prrx1 in PDGF-Induced Migration and Proliferation}

PDGF has been reported to increase the chemotaxis and proliferation of HSCs during fibrosis. ${ }^{26}$ Next, we used the gain- and loss-of-function approaches in combination with various assays to decipher the role of Prrxl as a modulator of PDGF-dependent processes. The silencing of Prrxl in primary HSCs isolated from rat and LX-2 significantly reduced their rate of migration both basally and after PDGF-BB treatment (Figure 5a). In addition, Prrxla obviously increased the migratory ability of LX-2 cells as compared with the cells transfected with Prrxlb plasmid or vectors (Figure 5b). Consecutively, we used the wound-healing assay to confirm the previous result. Consistent with the previous observations, Prrxl knockdown attenuated the migration of HSCs in response to PDGF-BB stimulation (Figure 5c). The results indicated that Prrxl might have a critical role in PDGF-dependent cell motility.

Furthermore, to assess whether Prrxl was involved in the regulation of PDGF-mediated proliferation, Prrx1 siRNAtreated HSC proliferation was assessed by CCK-8. Silencing the expression of Prrx1 did not affect the proliferation of HSCs (Supplementary Figure 2). These data demonstrated the vital role of Prrx1 in PDGF-mediated HSC migration and cell adhesion.

\section{Reduced Prrx1 Expression Increases HSC Adhesiveness to Fibronectin}

Cell adhesion and migration are critical pathological processes in liver fibrosis. ${ }^{27-29}$ To assess whether the defective migration of Prrx1 siRNA-transfected HSCs might result from altered cell adhesiveness, we performed a cell adhesion assay using fibronectin (FN) on HSCs. The knockdown of Prrxl expression increased the adhesion of HSCs to fibronectin in the presence or absence of PDGF-BB (Figure 5d and e). Our results indicated that Prrxl downregulation in HSCs significantly enhances their adhesiveness thereby impairing their ability of migration in response to PDGF-BB.
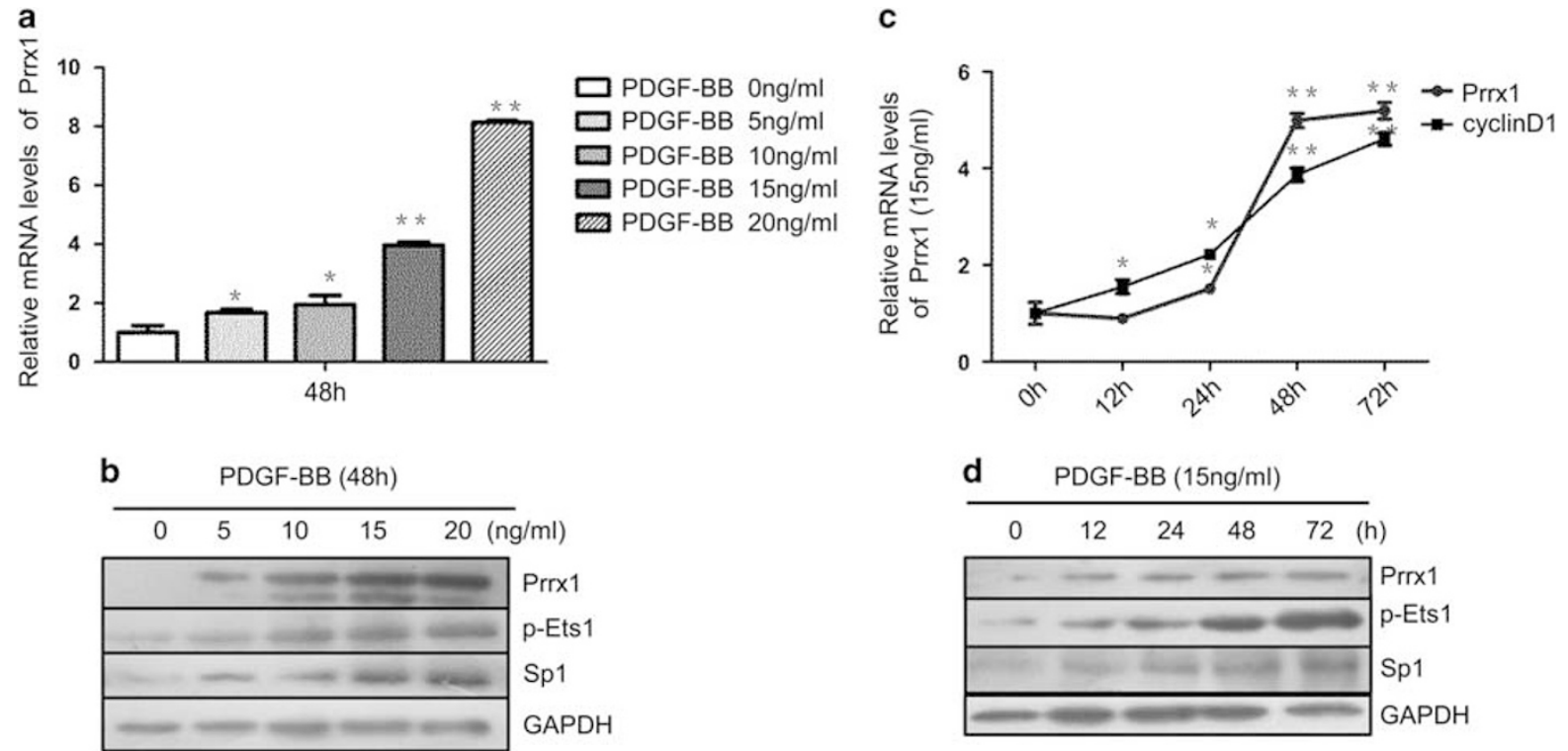

Figure 3 PDGF-BB-induced Prrx1 expression. (a and c) PDGF-BB induced Prrx1 level in a dose-dependent manner. LX-2 were serum-starved for $24 \mathrm{~h}$ and treated with the indicated concentrations of PDGF-BB $(0,5,10,15$, and $20 \mathrm{ng} / \mathrm{ml})$ with $1 \% \mathrm{FBS}$ for an additional $48 \mathrm{~h}$. RNA and protein were extracted from the cells. ( $\mathbf{b}$ and $\mathbf{d}$ ) PDGF-BB upregulated the Prrx1 expression in a time-dependent manner. LX-2 were serum-starved for $24 \mathrm{~h}$ and treated with $15 \mathrm{ng} / \mathrm{ml}$ PDGF at the indicated time points $(0,12,24,48$, and $72 \mathrm{~h}$ ) before the extraction of mRNA and protein. Prrx 1 mRNA level was quantified by real-time PCR; Prrx1, p-Ets1, and Sp1 protein levels were analyzed by western blot analysis. ${ }^{*} P<0.05,{ }^{* *} P<0.01$ vs control group by Student's $t$-test.

Figure 2 Prrx1 exerted its profibrotic role in HSCs. (a) Immunofluorescence co-staining of Prrx1 (red) and $a$-SMA (green) in the liver of human cirrhotic tissue. (b) Immunofluorescence staining of Prrx1 and a-SMA in freshly isolated (1 d) and activated (14 d) primary rat HSCs. Rat HSCs were in the quiescent state and had spontaneous blue-green fluorescence on the first day after isolation. 


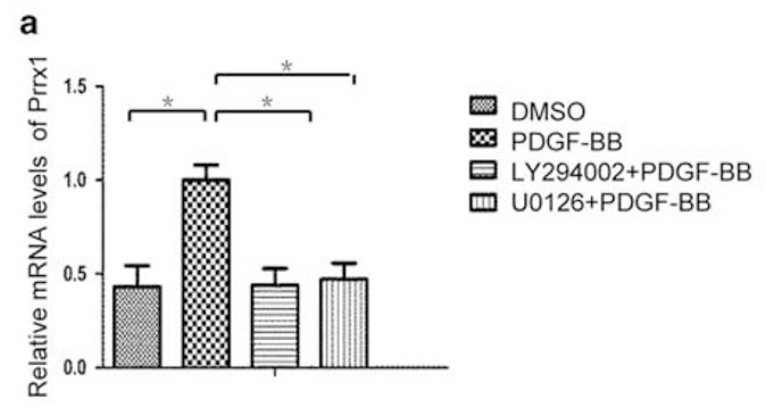

b
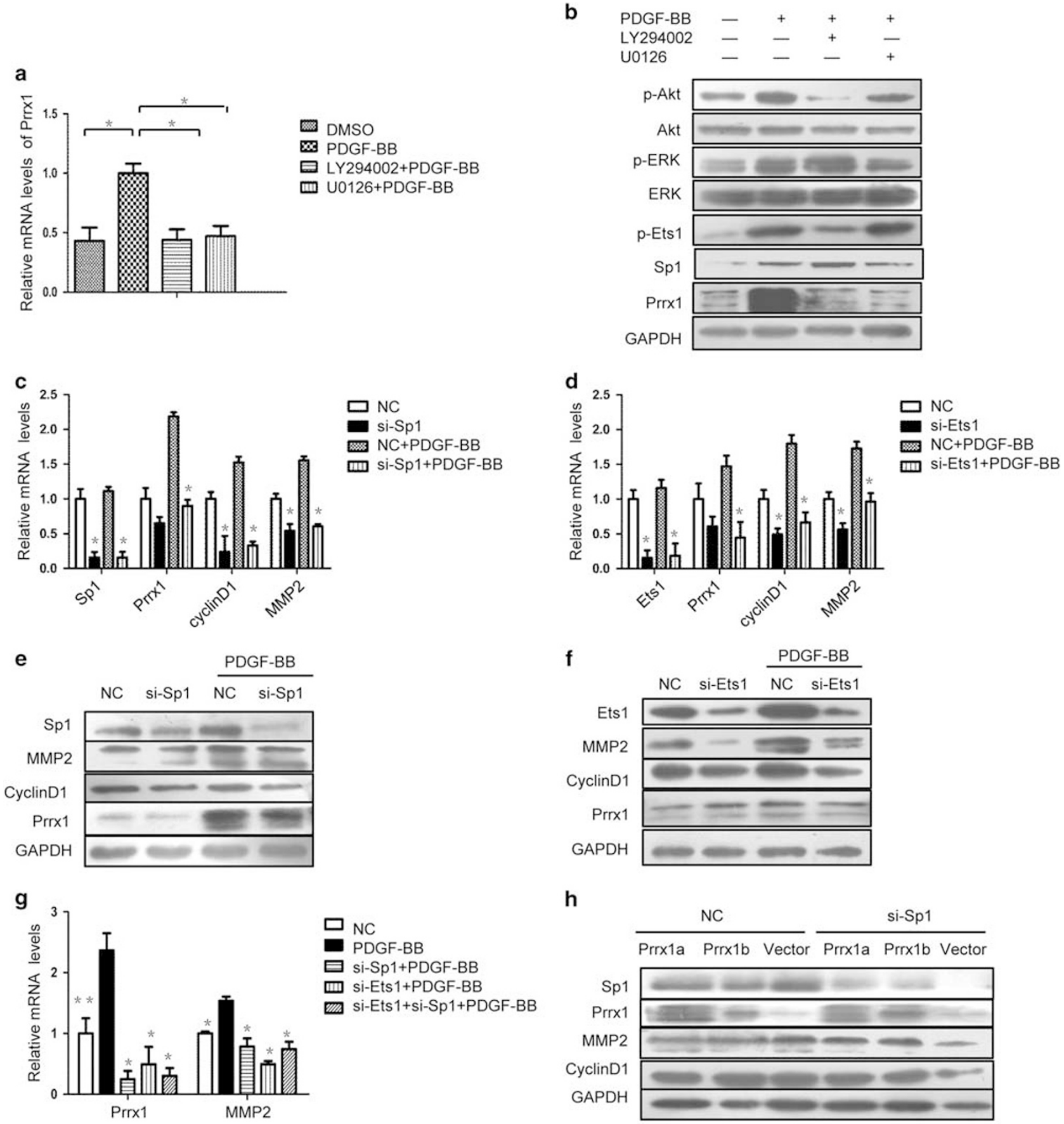

h

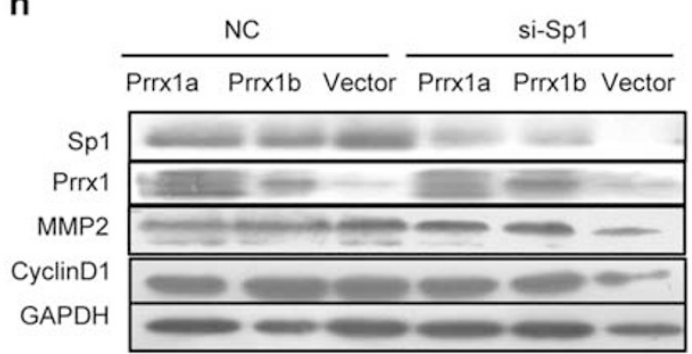

i

$\frac{\text { NC }}{\text { Prrx1a Prrx1b Vector }} \frac{\text { si-Ets1 }}{\text { Prrx1a Prrx1b Vector }}$

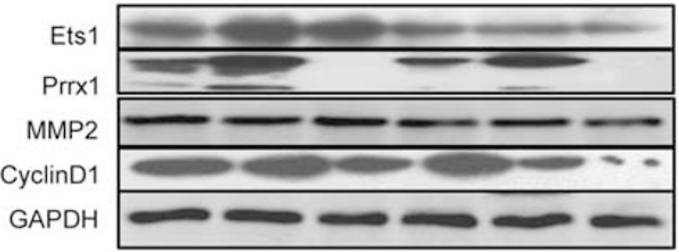




\section{Prrx1 Regulates PDGF-Dependent Process by Modulation of cyclinD1 and MMPs}

MMPs have a critical role in the degradation of ECM components; PDGF-BB induces MMP2- and MMP9mediated migration of HSCs. ${ }^{26,29-31}$ Therefore, we aspired to delineate whether Prrxl regulated the downstream effectors in the PDGF-dependent physiological process. Here, we found that in LX-2 cells, Prrxl siRNA prevented the PDGF-induced increase in the mRNA and protein levels of genes cyclinD1, MMP2, and MMP9 (Figure 6a-c). In

a

LX-2

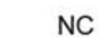

si-Prrx1

$\mathrm{NC}+\mathrm{PDGF}-\mathrm{BB}$

si-Prrx1 +PDGF-BB

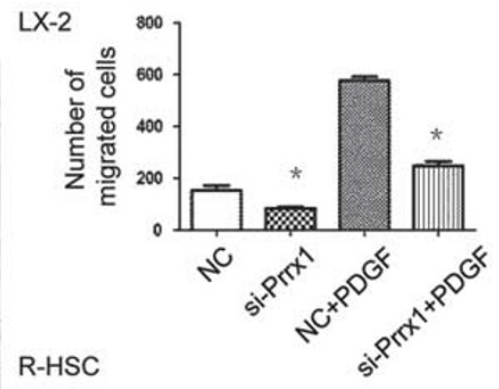

R-HSC

(48h)

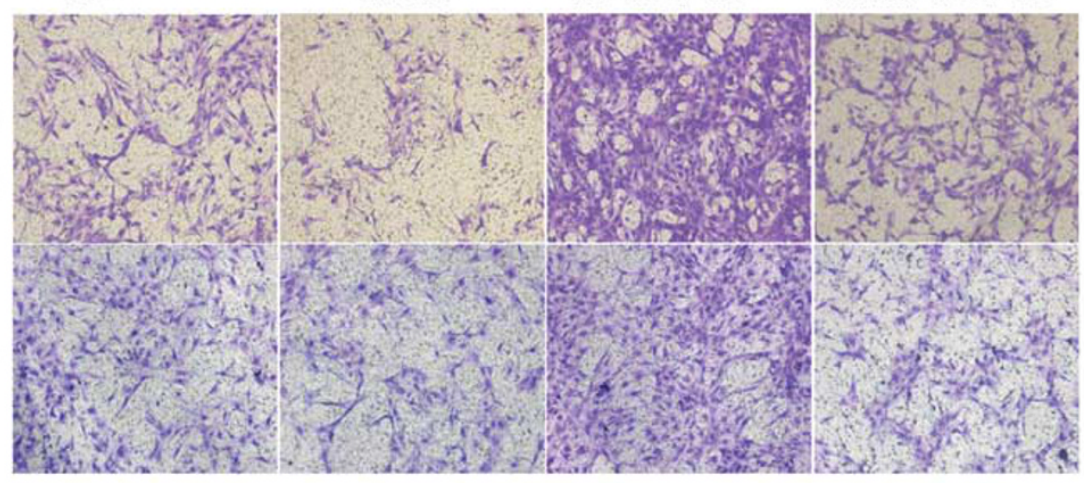

b

LX-2

(48h)

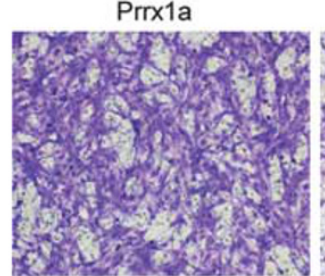

Prrx1b

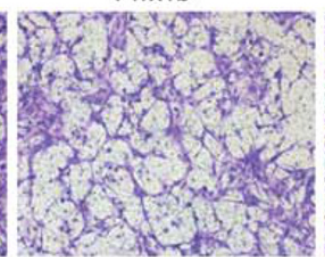

Vector

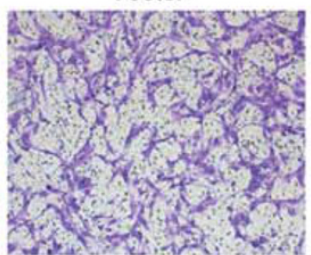

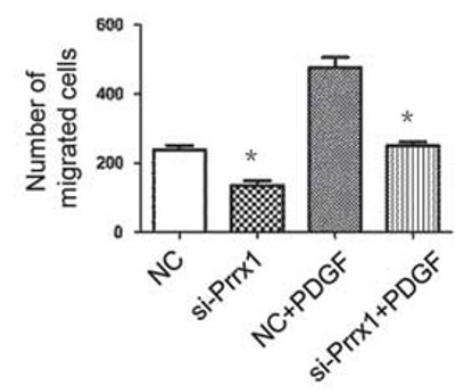

c
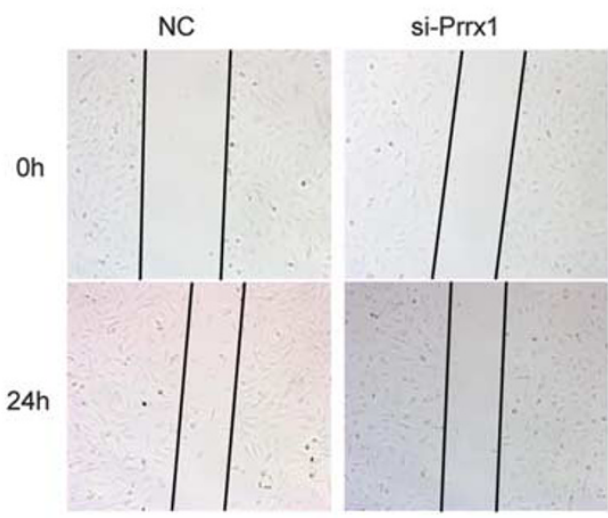

$\mathrm{NC}+\mathrm{PDGF}-\mathrm{BB}$

si-Prrx1 +PDGF-BB
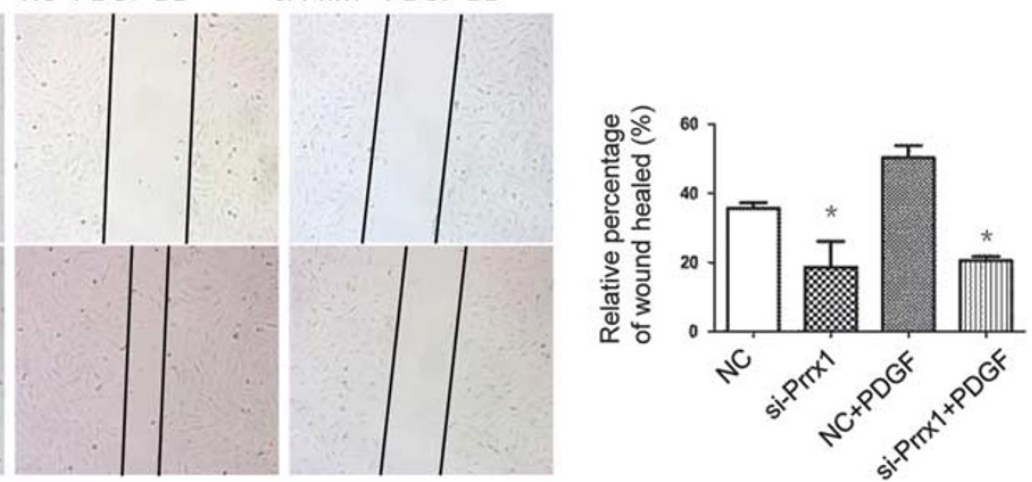

Figure 5 Prrx1 involved in PDGF-induced cell migration and knockdown of Prrx1 impaired cell adhesion to FN. (a and b) The migration rates of LX-2 cells and rat primary HSCs were measured after Prrx 1 expression was silenced or enhanced in the presence or absence of PDGF-BB (15 ng/ml) by transwell migration assays. The number of migrated cells were counted in four random fields. Scale bars $=100 \mu$ m. ${ }^{*} P<0.05$ siRNA $v s$ NC; Prrx 1 plasmid vs vector. (c) Representative images of the wound-healing assay with LX-2 cells. LX-2 cells were transfected with Prrx1 siRNA or NC in response to PDGFBB stimulation. Cell migration was quantified as a percentage of wound-healed area. (d and e) Prrx 1 expression was silenced in the presence or absence of PDGF-BB (15 ng/ml), representative images of adhesive cells stained with DAPI. The adhesion ability of HSCs was assessed by CCK-8. *P < 0.05 vs NC or PDGF-BB.

Figure 4 PDGF-BB promoted Prrx1 expression by activating ERK/Sp1 and Akt/p-Ets1 in HSCs. (a and b) LX-2 cells were treated with ERK inhibitor (U0126, $25 \mu \mathrm{M})$ and PI3K inhibitor (LY294002, $25 \mu \mathrm{M})$ for $1 \mathrm{~h}$ before PDGF-BB stimulation (15 ng/ml) for an additional $48 \mathrm{~h}$. ERK and Akt signals, p-Ets1, Sp1, and Prrx1 levels were examined by western blot. Prrx1 mRNA level was detected by real-time PCR. *P<0.05 vs PDGF-BB. (c and e) LX-2 cells were transfected with Sp1 siRNA or control siRNA in the presence or absence of PDGF-BB (15 ng/ml) for $48 \mathrm{~h}$. Sp1, Prrx1, MMP2, and cyclinD1 levels were detected by real-time PCR and western blot. ( $\mathbf{d}$ and $\mathbf{f}$ ) LX-2 cells were transfected with Ets1 siRNA or control siRNA in the presence or absence of PDGFBB (15 ng/ml) for 48 h. p-Ets1, Prrx1, MMP2, and cyclinD1 levels were detected by real-time PCR and Western blot. ${ }^{*} P<0.05$, ${ }^{* *} P<0.01$. (g) LX-2 cells were transfected with Ets1 siRNA, Sp1 siRNA, Ets1 siRNA+Sp1 siRNA, or control siRNA in the presence of PDGF-BB (15 ng/ml) for 48 h. Prrx1 mRNA level was detected by real-time PCR. ${ }^{*} P<0.05,{ }^{*} P<0.01$. (h and i) LX-2 cells were transfected with Sp1 siRNA (h) or Ets1 siRNA (i). After 24 h, the cells were transfected with Prrx1a, Prrx1b, and control vector plasmids for an additional $48 \mathrm{~h}$, respectively. Western blot analysis was used to examine the expression of Ets1, Sp1, Prrx1, MMP2, and cyclinD1. 
d
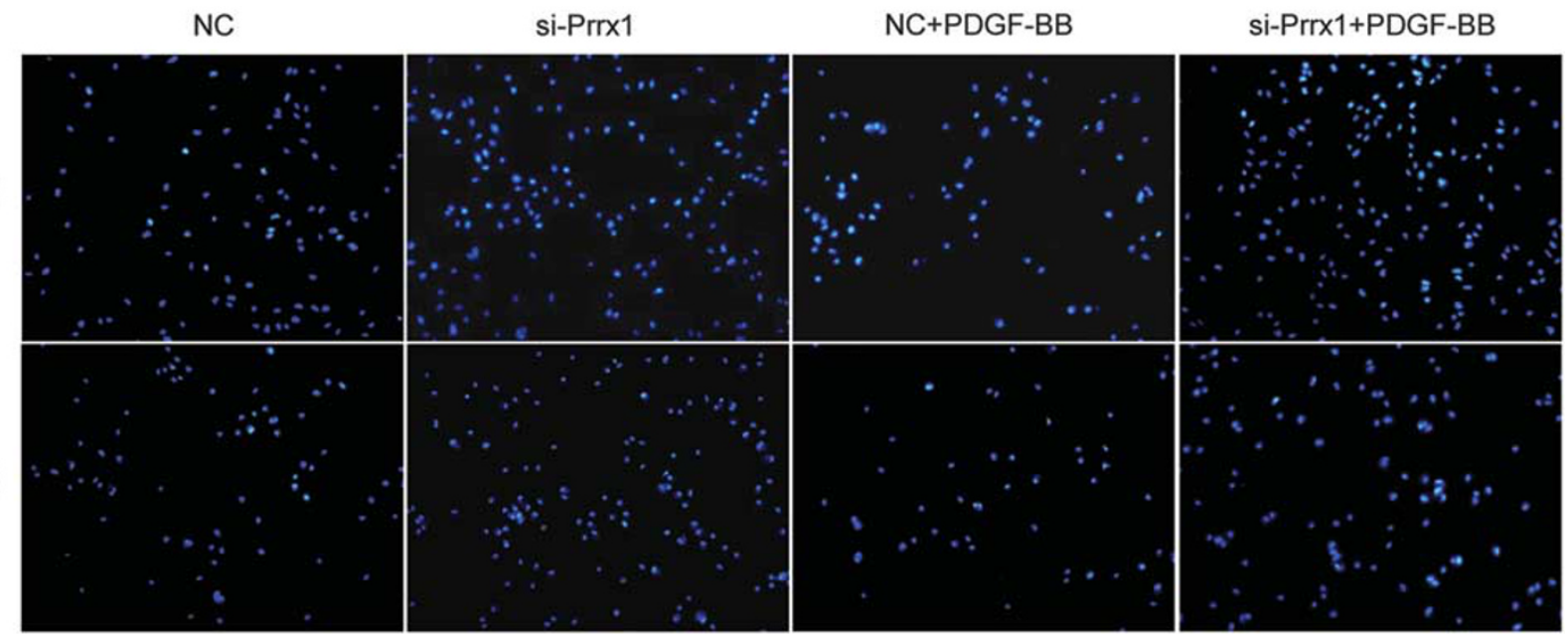

e

LX-2

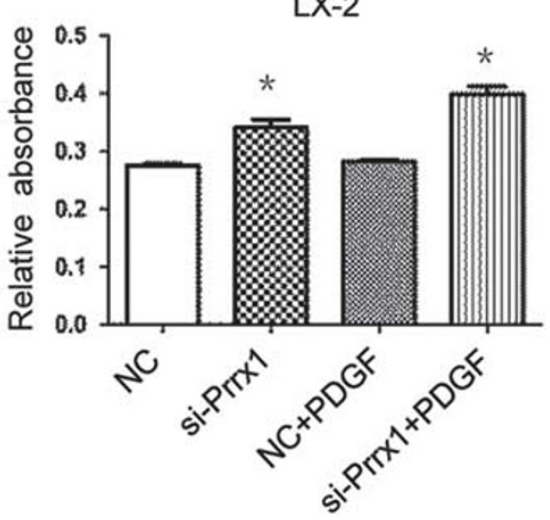

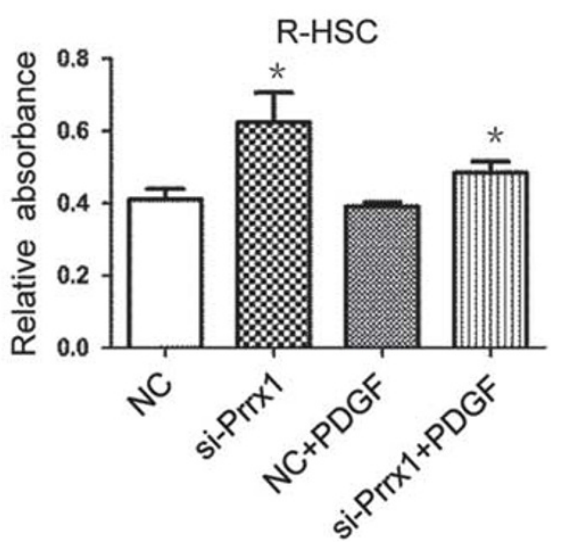

Figure 5 Continued.

addition, LX-2 cells transfected with Prrx1 plasmids and vector were then subjected to the treatment with PDGF-BB or vehicle, respectively. The results showed that the enforced expression of Prrx1 could upregulate MMP2 and MMP9 mRNA levels both at baseline and after PDGF-BB treatment. However, any distinct increase in the expressions of MMP2 and MMP9 was not found as compared with the LX-2 cells transfected with Prrx1 plasmids between vehicle and PDGFBB stimulation group (Figure 6d). These data indicated that Prrx1 may be the key factor for PDGF-mediated MMP2/ MMP9 upregulation. Next, we explored the relationship between Prrx1, Ets1, and Sp1, as all of them could regulate expression of the downsteam molecules MMP2. Through double immunofluorescence staining of Prrxl, Ets1, and Sp1 in LX-2, we could find that they were co-localized in the nucleus of LX-2 cells (Figure 6e). Besides, LX-2 cells were transfected with plasmid encoding Prrxl-Flag, then we investigated whether Prrx1 could work together with Ets1 or Sp1 by coimmunoprecipitation. The result showed that anti-Flag antibody co-immunoprecipitated complexes containing Sp1 and Ets1, and Prrx1 rabbit antibody identified the expression of Prrxl, which indicated Prrxl could have interacted with Sp1 and Ets1 (Figure 6f and Supplementary Figure 3).

\section{Knockdown of Prrx1 Attenuates TAA-Induced Liver Fibrosis in Rat}

To confirm the role of Prrxl on fibrogenesis in vivo, we silenced Prrx 1 expression in a rat model of TAA-induced liver fibrosis. After tail-vein injection of AdshPrrxl, we observed that Prrxl silencing resulted in reduced liver fibrosis by hematoxylin and eosin (H\&E), Masson's trichrome, $\alpha$-SMA expression (Figure $7 \mathrm{a}$ and $\mathrm{b}$ and Supplementary Figure 4), and hydroxyproline contents (Figure 7c). In addition, the AdshPrrxl administration significantly attenuated the liver damage as shown by decreased ALT and AST serum levels (Figure 7d). Furthermore, collagen $1 \alpha 1, \alpha$-SMA, and MMP2 levels were significantly reduced after AdshPrrxl treatment (Figure $7 \mathrm{e}$ and $\mathrm{f}$ ). Taken together, our results showed that Prrxl silencing interferes with the development of TAAinduced liver fibrosis.

\section{DISCUSSION}

Previous studies have demonstrated that the serum level of PDGF-BB increased in patients with fibrosis of various etiologies and fibrotic livers in the mouse model. ${ }^{4,32}$ PDGF is a principal growth factor that potently drives the proliferation and migration of HSCs. ${ }^{33}$ In recent decades, intensive 
a

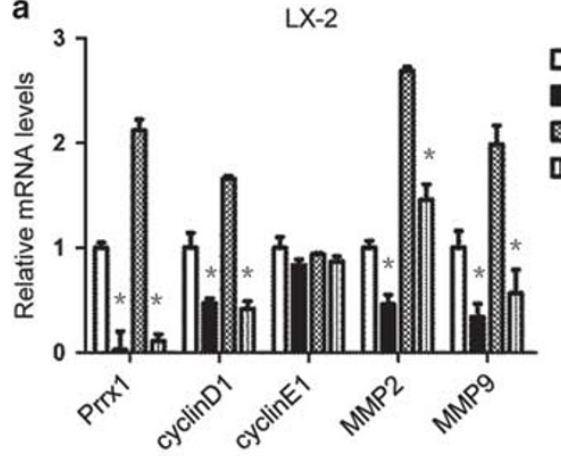

c
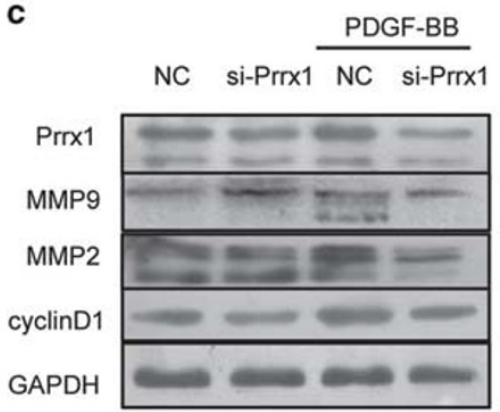

NC

si-Prrx1

NC+PDGF-BB

si-Prrx1+PDGF-BB b

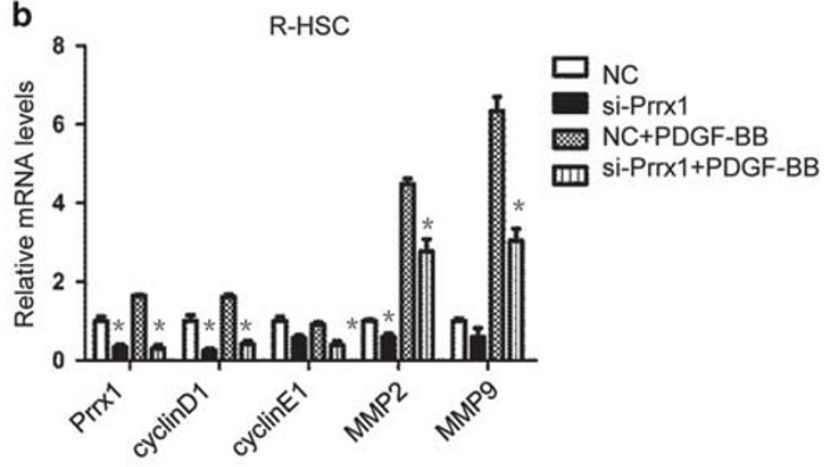

d

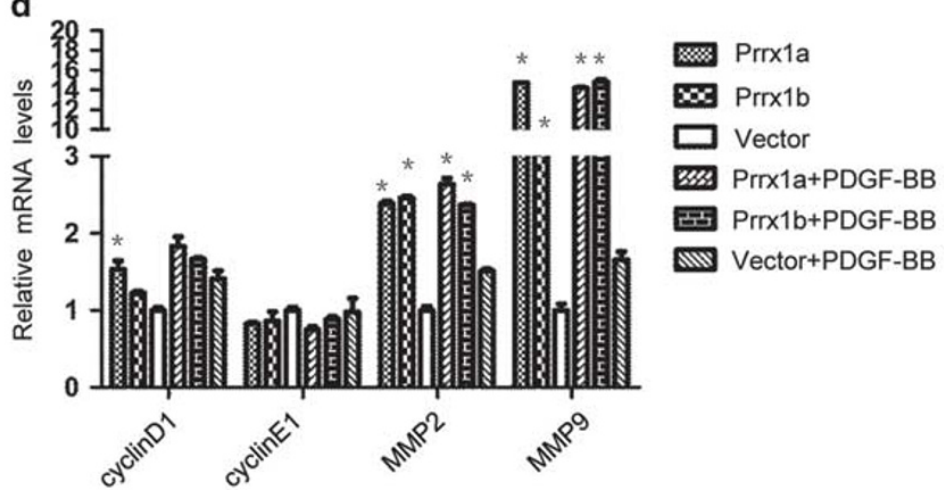

e

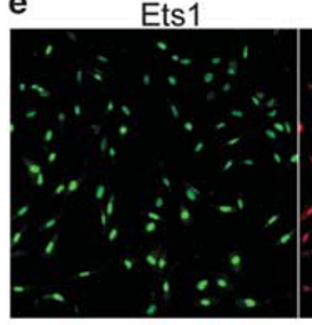

$\mathrm{Sp} 1$

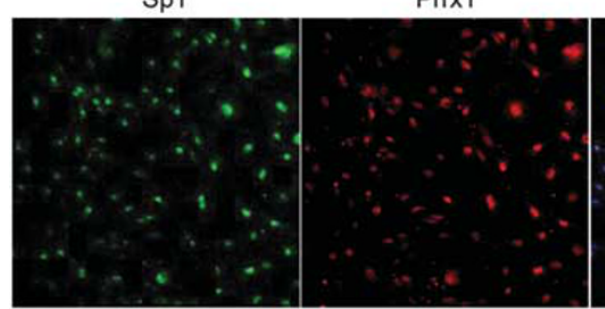

Prrx1

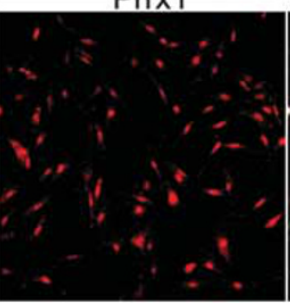

Prrx1

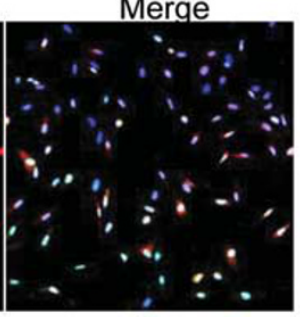

Merge

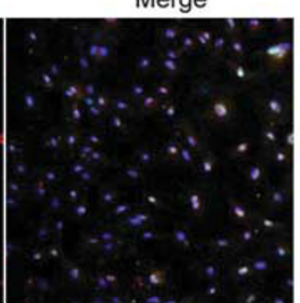

f

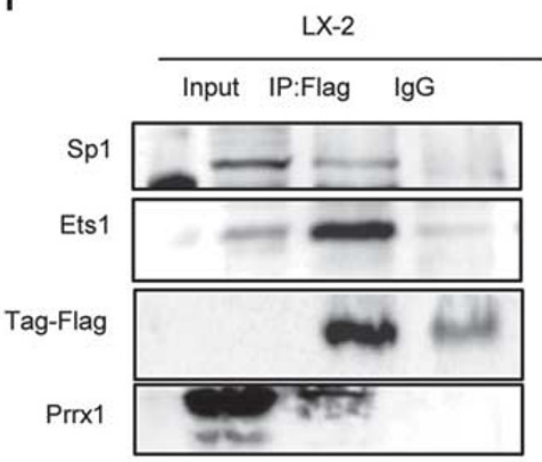

Figure 6 MMP2 and MMP9 were downstream targets of Prrx1 in PDGF-treated cells. (a and b) Prrx1 silencing in LX-2 cells and rat primary HSCs decreased the PDGF-induced upregulation of specific mRNAs. ${ }^{*} P<0.05$ vs NC or PDGF-BB. (c) LX-2 cells were transfected with Prrx1 siRNA in the presence or absence of PDGF-BB $(15 \mathrm{ng} / \mathrm{ml})$; western blot was used to examine the protein levels of Prrx1, MMP2, and cyclinD1. (d) LX-2 cells were transfected with Prrx 1 or vector for $24 \mathrm{~h}$, then stimulated with PDGF-BB $(15 \mathrm{ng} / \mathrm{ml})$ for an additional $48 \mathrm{~h}$. The mRNA levels were tested by real-time PCR. ${ }^{*} P<0.05$ vs vector or PDGF+vector. (e) Immunofluorescence co-staining of Prrx1, Ets1, and Sp1 in LX-2 cells. (f) LX-2 cells were transfected with plasmids encoding Flag-Prrx1. After $48 \mathrm{~h}$, cell lysates were prepared and used for co-immunoprecipitation.

research is focused on new therapies targeting the PDGF pathways; however, due to varied individual basis and low targeting efficiency, limited therapeutic advances have been made. ${ }^{34,35}$ The current study, for the first time, demonstrates Prrx1 as a potential target in liver fibrosis that could regulate the PDGF-induced chemotaxis. Moreover, Prrx1 is stimulated by PDGF in a dose- and time-dependent manner in HSCs, which indicates the correlation between Prrxl and PDGF stimulation. This phenomenon could also explain why Prrx 1 is strongly expressed in HSCs in the fibrotic liver while being nearly undetectable in healthy liver tissue.

Prrx1, pair-related homeobox transcription factor, is upregulated 10-50-fold in the fibrotic liver and activated HSCs. ${ }^{36}$ Consistent with the previous studies, our results 
showed that Prrxl expression was dramatically elevated in TAA- and $\mathrm{CCl}_{4}$-induced fibrosis. Despite the role of Prrx1 in HSC activation, we used complementary approaches including the gain- and loss-of-function to demonstrate the vital role of Prrx1 in the proliferation, motility, and cell adhesion of HSCs. We confirmed that the knockdown of a

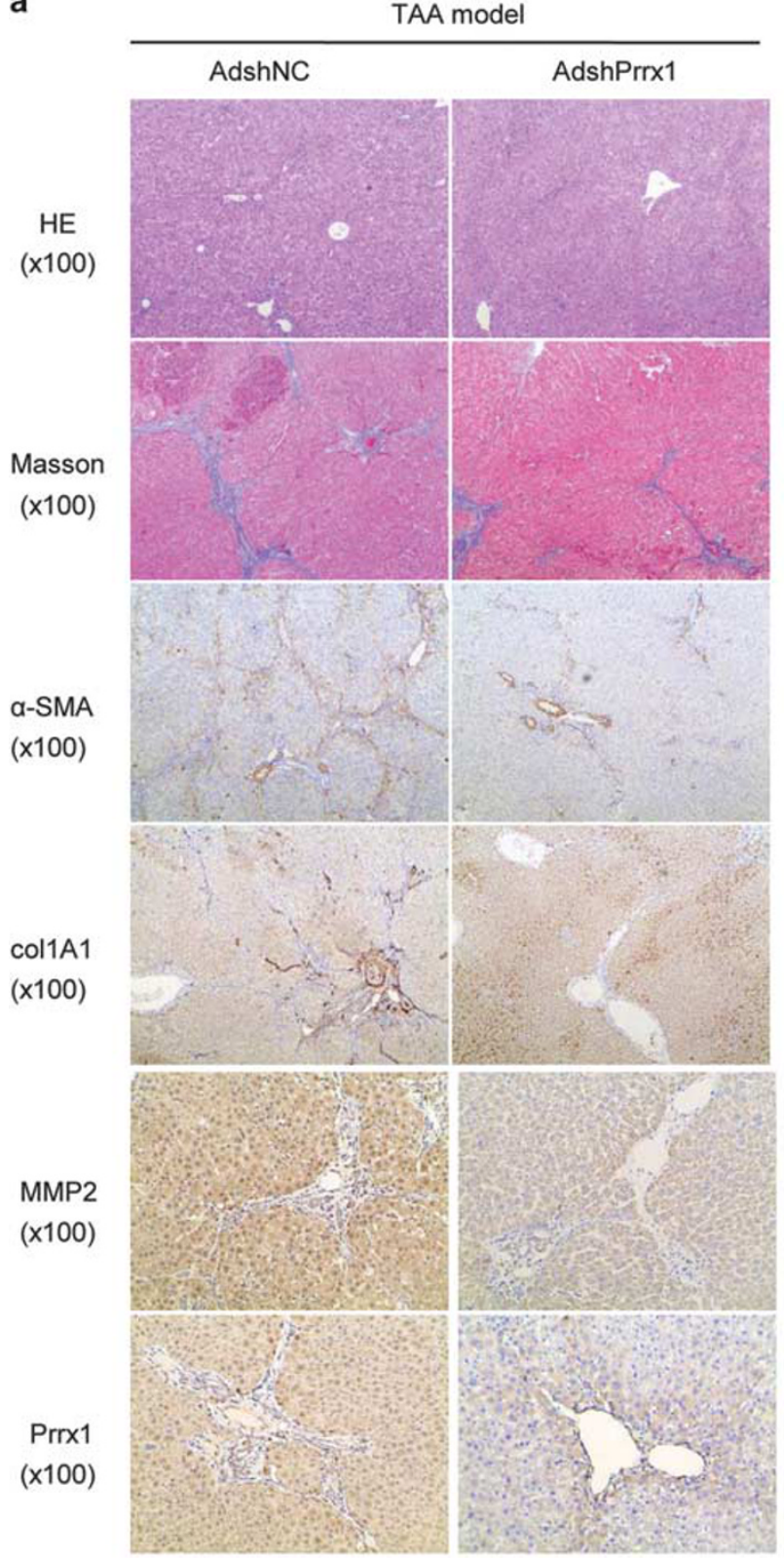

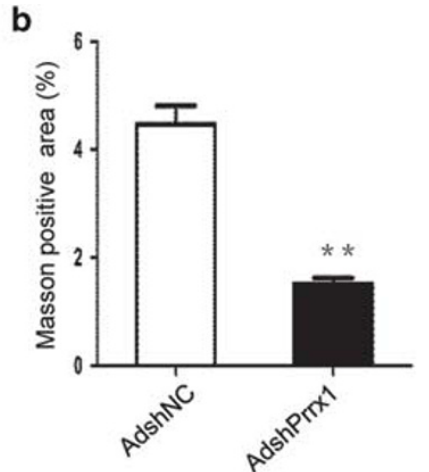
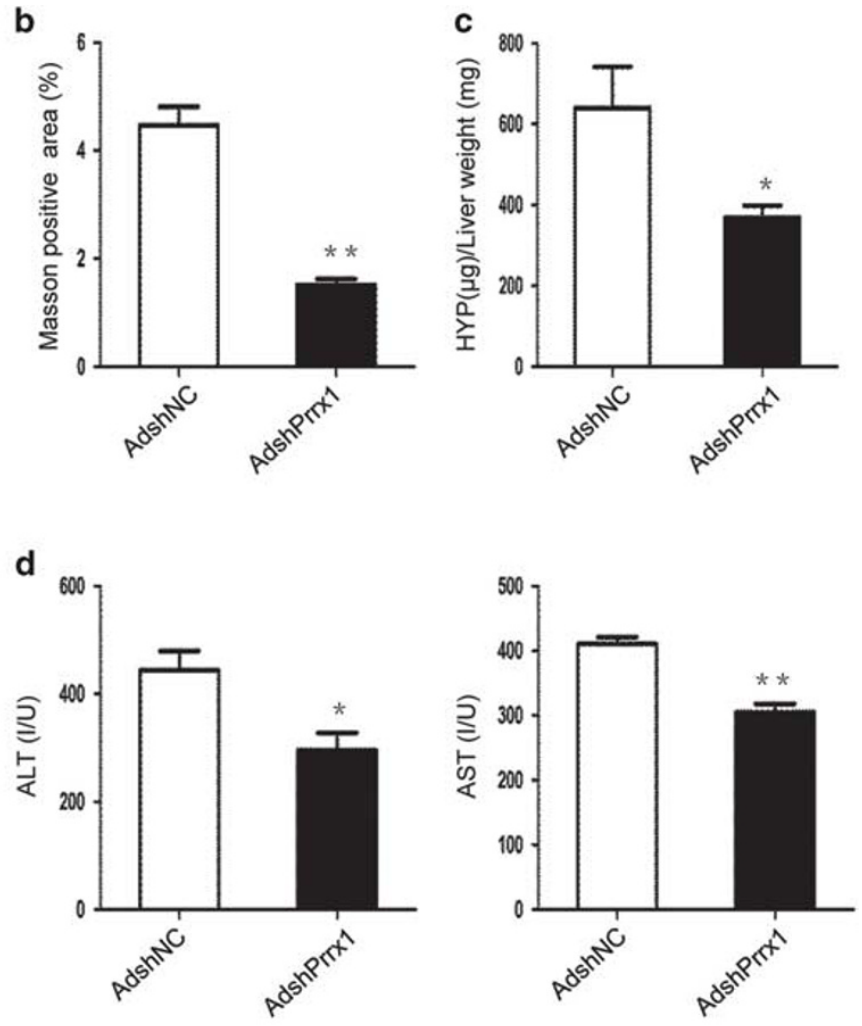

e

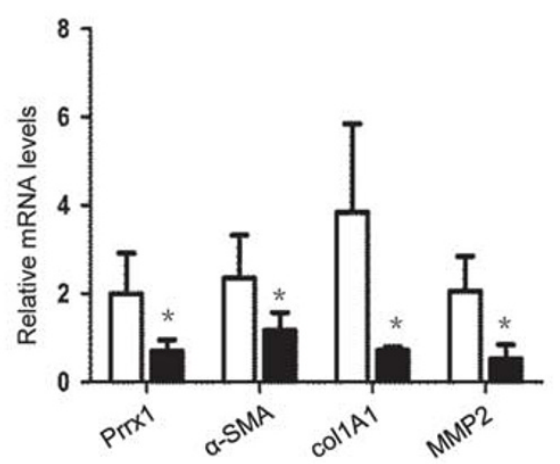

AdshNC

AdshPrrx1

f

TAA model

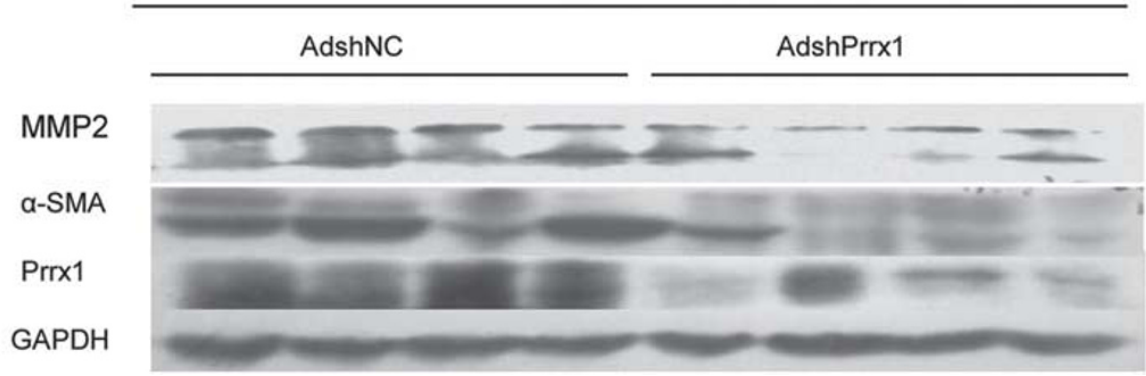


Prrx1 impaired the motility of HSCs and increased cell adhesion in the presence or absence of PDGF. Conversely, the overexpression of Prrx1 enforced the migration of HSCs. This phenomenon could be further strengthened by the recent evidence that Prrx1 is an EMT inducer conferring migratory and invasive properties in pancreatic cancer and breast cancer cells. ${ }^{16,18}$ The microarray data from another study showed that silencing Prrxl in adult neural stem cells led to the upregulation of the cell adhesion molecules involved in the HSC-associated phenotypes. ${ }^{15}$ Furthermore, another report showed that in lung mesodermal cells, the expression of Prrx1 was essential for the aggregation of disorganized mesenchymal cells into an organized monolayer of vascular endothelium by cell-to-cell contact, ${ }^{37}$ which indicates the putative modulation of Prrx1 on cell adhesion. Collectively, these results indicated that Prrx1 could regulate the various cellular processes, such as synthesis of collagen, cell migration, and cell adhesion.

ERK and PI3K-Akt signaling pathways are known to be involved in PDGF-stimulated mitogenesis, migration, and chemotaxis. ${ }^{25}$ Our results indicated that ERK and PI3K-Akt cascades were vital for PDGF-induced activation of Prrx1 since the specific blockade of ERK or PI3K resulted in a significant reduction in the level of Prrx1 in the presence of PDGF. Furthermore, we demonstrated that the transcription factor Sp1, acting as a downstream signaling molecular of ERK, had a major role in the regulation of Prrxl gene expression in HSCs, which is also supported by several lines of evidence. First, the inhibition of the ERK1/2 signaling pathway abolished the PDGF-BB-induced Sp1 and Prrx1 expressions. Second, the knockdown of $\mathrm{Sp} 1$ prevented the PDGF-BB-induced activation of the Prrx1 expression. Next, the stimulation with PDGF-BB enhanced the level of Sp1 protein similar to that of the Prrxl level. Finally, the restoration of Prrx1 expression after silencing Sp1 recovered the expression of the downstream effectors, such as cyclinD1 and MMP2. Altogether, these findings, for the first time, revealed Sp1 as a downstream target of ERK1/2 in PDGFstimulated HSC-regulated Prrxl expression. This observation was in agreement with the previous study describing Sp1 activation as critical for PDGF-BB-mediated phenotypic modulation of smooth muscle. ${ }^{38}$ Similarly, we confirmed that PDGF increased the Prrx1 transcription via PI3Kmediated activation of $\mathrm{p}$-Ets 1 ; this was similar to the previous study showing Ets1 as a downstream molecule of PI3K regulated by PDGF. ${ }^{25}$ Compared with targeting Sp1 or Ets1 separately, LX-2 cells did not further decrease Prrx1 mRNA level when both $\mathrm{Sp} 1$ and Ets siRNAs are expressed together. It could be possible that the two pathways have a synergistic effect on Prrx1 expression. However, the precise mechanism underlying the activation of transcription factors Sp1 and Ets1 that regulate the expression of Prrx1 in HSCs necessitates further investigation.

The present study also provides an in vivo evidence that adenovirus-mediated knockdown of Prrxl in TAA-induced liver injury model reduces the liver damage, inflammation, and development of liver fibrosis. Compared with the AdGFP injection, Prrxl silencing significantly reduces the expression of HSC activation markers and attenuates the ECM deposition and liver function, which indicates that Prrxl could attenuate liver fibrosis by deactivating the HSCs. This result was complementary to a previous study describing that adenovirus-mediated delivery of Prrxla in mouse augmented collagen and the expression of $\alpha$-SMA. ${ }^{20}$ Moreover, in agreement with the results that Prrx1 upregulated MMP2, MMP9, and cyclinD1 in vitro, the silencing of the gene leads to less-activated HSCs as presented in a fibrotic scar in vivo. This manifestation may be associated with the decrease in the motility and proliferation of HSCs and degradation of ECM. Liver fibrosis is always associated with enhanced expression of matrix protein and production of MMPs. ${ }^{26}$ The MMPs could proteolytically degrade $\mathrm{ECM},{ }^{39}$ as well as, contribute to cell adhesion and migration. ${ }^{27,29,31,40}$ Therefore, Prrx1 mediated the upregulation of MMP2 and MMP9 expression that could decrease cell adhesion ability and promote the migration of HSCs. However, other underlying molecular and cellular mechanisms require further investigation.

In summary, we have identified Prrx1 as a novel valuable target in liver fibrosis, which had a major role in PDGF-induced migration and chemotaxis. Moreover, PDGF-BB via ERK-Sp1 and PI3K/Akt/Ets1 signaling increased Prrxl expression in a dose- and time-dependent manner. Finally, our data also demonstrated that interference in Prrxl expression could attenuate liver fibrosis development, partially by the repression of $\alpha$-SMA, col1A1, and MMP2. Therefore, the novel regulatory mechanisms discovered about Prrx1 are speculated to provide an in-depth insight about PDGF signaling and a potential therapeutic target in liver fibrosis.

Supplementary Information accompanies the paper on the Laboratory Investigation website (http://www.laboratoryinvestigation.org)

Figure 7 Knockdown of Prrx1 suppressed the TAA-induced hepatic fibrosis in rats. (a) AdshPrrx1 or AdshNC was injected into rats 6 weeks after treatment with TAA. Subsequently, after 2 weeks, the expression of COL1A1, $a-S M A, M M P 2$, and Prrx 1 in the fibrotic livers was analyzed by immunohistochemistry. HE and Masson's trichrome staining were used to examine the pathological alterations and collagen deposition. (b) Semiquantitative analysis of Masson's trichrome staining in the fibrotic livers from AdshPrrx1- or AdshNC-treated rats ( $n=6$ rats in each group). (c) Hydroxyproline level in livers from AdshPrrx1- or AdshNC-treated rats in the TAA-induced fibrotic model $(n=6)$. (d) Serum biochemical parameters, ALT and AST, were analyzed from AdshPrrx1- or AdshNC-treated rats in the TAA-induced fibrotic model $(n=4)$. (e and $\mathbf{f})$ mRNA and protein levels of COL1A1, $a$-SMA, MMP2, and Prrx1 in the livers were detected by real-time PCR $(n=5)$ and western blot $(n=4)$, respectively. GAPDH was used as an endogenous control. ${ }^{*} P<0.05,{ }^{* *} P<0.01$ by Student's $t$-test. 


\section{ACKNOWLEDGMENTS}

This study was supported by the National Natural Science Foundation of China (No. 81270507, No. 81572419).

\section{DISCLOSURE/CONFLICT OF INTEREST}

The authors declare no conflict of interest.

1. Schuppan D, Afdhal NH. Liver cirrhosis. Lancet 2008;371:838-851.

2. Hernandez-Gea V, Friedman SL. Pathogenesis of liver fibrosis. Annu Rev Pathol 2011;6:425-456.

3. Seki E, Schwabe RF. Hepatic inflammation and fibrosis: functional links and key pathways. Hepatology 2015;61:1066-1079.

4. Yoshida S, Ikenaga N, Liu SB, et al. Extrahepatic platelet-derived growth factor-beta, delivered by platelets, promotes activation of hepatic stellate cells and biliary fibrosis in mice. Gastroenterology 2014;147:1378-1392.

5. Hayes BJ, Riehle KJ, Shimizu-Albergine M, et al. Activation of plateletderived growth factor receptor alpha contributes to liver fibrosis. PLoS ONE 2014;9:e92925.

6. Wright $\mathrm{JH}$, Johnson $\mathrm{MM}$, Shimizu-Albergine $\mathrm{M}$, et al. Paracrine activation of hepatic stellate cells in platelet-derived growth factor $\mathrm{C}$ transgenic mice: evidence for stromal induction of hepatocellular carcinoma. Int J Cancer 2014;134:778-788.

7. Soriano P. Abnormal kidney development and hematological disorders in PDGF beta-receptor mutant mice. Genes Dev 1994;8:1888-1896.

8. Lindblom P, Gerhardt H, Liebner S, et al. Endothelial PDGF-B retention is required for proper investment of pericytes in the microvessel wall. Genes Dev 2003;17:1835-1840.

9. Kocabayoglu P, Lade A, Lee YA, et al. beta-PDGF receptor expressed by hepatic stellate cells regulates fibrosis in murine liver injury, but not carcinogenesis. J Hepatol 2015;65:1417-1417.

10. Grueneberg DA, Natesan $S$, Alexandre $C$, et al. Human and drosophila homeodomain proteins that enhance the DNA-binding activity of serum response factor. Science 1992;257:1089-1095.

11. Simon KJ, Grueneberg DA, Gilman M. Protein and DNA contact surfaces that mediate the selective action of the Phox 1 homeodomain at the c-fos serum response element. Mol Cell Biol 1997;17:6653-6662.

12. ten Berge D, Brouwer A, Korving J, et al. Prx1 and Prx2 are upstream regulators of sonic hedgehog and control cell proliferation during mandibular arch morphogenesis. Development 2001;128:2929-2938.

13. Dasouki $M$, Andrews $B$, Parimi $P$, et al. Recurrent agnathia-otocephaly caused by DNA replication slippage in PRRX1. Am J Med Genet A 2013;161:803-808.

14. Martin JF, Olson EN. Identification of a prx1 limb enhancer. Genesis 2000;26:225-229.

15. Shimozaki K, Clemenson GD Jr, Gage FH. Paired related homeobox protein 1 is a regulator of stemness in adult neural stem/ progenitor cells. J Neurosci 2013;33:4066-4075.

16. Reichert M, Takano S, von Burstin J, et al. The Prrx1 homeodomain transcription factor plays a central role in pancreatic regeneration and carcinogenesis. Genes Dev 2013;27:288-300.

17. McKean DM, Sisbarro L, llic D, et al. FAK induces expression of Prx1 to promote tenascin-C-dependent fibroblast migration. J Cell Biol 2003;161:393-402.

18. Ocana $\mathrm{OH}$, Corcoles $\mathrm{R}$, Fabra $\mathrm{A}$, et al. Metastatic colonization requires the repression of the epithelial-mesenchymal transition inducer Prrx1. Cancer Cell 2012;22:709-724.

19. Fritz D, Stefanovic B. RNA-binding protein RBMS3 is expressed in activated hepatic stellate cells and liver fibrosis and increases expression of transcription factor Prx1. J Mol Biol 2007;371:585-595.

20. Jiang F, Stefanovic B. Homeobox gene Prx1 is expressed in activated hepatic stellate cells and transactivates collagen alpha1(I) promoter. Exp Biol Med (Maywood) 2008;233:286-296.
21. Lin J, Chen A. Activation of peroxisome proliferator-activated receptorgamma by curcumin blocks the signaling pathways for PDGF and EGF in hepatic stellate cells. Lab Invest 2008;88:529-540.

22. Zhang F, Kong D, Chen L, et al. Peroxisome proliferator-activated receptor-gamma interrupts angiogenic signal transduction by transrepression of platelet-derived growth factor-beta receptor in hepatic stellate cells. J Cell Sci 2014;127:305-314.

23. Woodhoo A, Iruarrizaga-Lejarreta M, Beraza N, et al. Human antigen R contributes to hepatic stellate cell activation and liver fibrosis. Hepatology 2012;56:1870-1882.

24. Deaton RA, Gan Q, Owens GK. Sp1-dependent activation of KLF4 is required for PDGF-BB-induced phenotypic modulation of smooth muscle. Am J Physiol Heart Circ Physiol 2009;296:H1027-H1037.

25. Jinnin $M$, Ihn $H$, Asano $Y$, et al. Platelet derived growth factor induced tenascin-C transcription is phosphoinositide 3-kinase/Akt-dependent and mediated by Ets family transcription factors. J Cell Physiol 2006;206:718-727.

26. Yang $C$, Zeisberg $M$, Mosterman B, et al. Liver fibrosis: insights into migration of hepatic stellate cells in response to extracellular matrix and growth factors. Gastroenterology 2003;124:147-159.

27. Atorrasagasti $C$, Aquino JB, Hofman L, et al. SPARC downregulation attenuates the profibrogenic response of hepatic stellate cells induced by TGF-beta1 and PDGF. Am J Physiol Gastrointest Liver Physiol 2011;300:G739-G748.

28. Cao S, Yaqoob U, Das A, et al. Neuropilin-1 promotes cirrhosis of the rodent and human liver by enhancing PDGF/TGF-beta signaling in hepatic stellate cells. J Clin Invest 2010;120:2379-2394.

29. Wang XM, Yu DM, McCaughan GW, et al. Fibroblast activation protein increases apoptosis, cell adhesion, and migration by the LX-2 human stellate cell line. Hepatology 2005;42:935-945.

30. Naito T, Tanihata $\mathrm{Y}$, Nishimura $\mathrm{H}$, et al. Expression of matrix metalloproteinase-9 associated with ets-1 proto-oncogene in rat tubulointerstitial cells. Nephrol Dial Transplant 2005;20:2333-2348.

31. Galli A, Svegliati-Baroni G, Ceni E, et al. Oxidative stress stimulates proliferation and invasiveness of hepatic stellate cells via a MMP2mediated mechanism. Hepatology 2005;41:1074-1084.

32. Wilhelm A, Aldridge V, Haldar D, et al. CD248/endosialin critically regulates hepatic stellate cell proliferation during chronic liver injury via a PDGF-regulated mechanism. Gut 2015;65:1175-1185.

33. Kong $\mathrm{X}$, Horiguchi $\mathrm{N}$, Mori $\mathrm{M}$, et al. Cytokines and STATs in liver fibrosis. Front Physiol 2012;3:69.

34. Mehal WZ, Schuppan D. Antifibrotic therapies in the liver. Semin Liver Dis 2015;35:184-198.

35. Popov Y, Schuppan D. Targeting liver fibrosis: strategies for development and validation of antifibrotic therapies. Hepatology 2009;50:1294-1306.

36. Jiang F, Parsons CJ, Stefanovic B. Gene expression profile of quiescent and activated rat hepatic stellate cells implicates Wnt signaling pathway in activation. J Hepatol 2006;45:401-409.

37. Dettman RW, Steinhorn RH. Connecting the cells: vascular differentiation via homeobox genes and extracellular matrix in the distal lung. Circ Res 2004;94:1406-1407.

38. Azahri NS, Di Bartolo BA, Khachigian LM, et al. Sp1, acetylated histone3 and p300 regulate TRAIL transcription: mechanisms of PDGF-BBmediated VSMC proliferation and migration. J Cell Biochem 2012;113: 2597-2606.

39. Xu L, Hui AY, Albanis $E$, et al. Human hepatic stellate cell lines, LX-1 and LX-2: new tools for analysis of hepatic fibrosis. Gut 2005;54:142-151.

40. Wang $H$, Zhu $Y$, Zhao $M$, et al. miRNA-29c suppresses lung cancer cell adhesion to extracellular matrix and metastasis by targeting integrin beta1 and matrix metalloproteinase2 (MMP2). PLoS ONE 2013;8: e70192. 